Case Report

\title{
Successful Resection of Isolated Para-Aortic Lymph Node Recurrence from Advanced Sigmoid Colon Cancer following 156 Courses of FOLFIRI Regimen
}

\author{
Kaoru Takeshima, Kazuo Yamafuji, Atsunori Asami, Hideo Baba, Nobuhiko Okamoto, \\ Hidena Takahashi, Chisato Takagi, and Kiyoshi Kubochi \\ Department of Surgery, Saitama City Hospital, Saitama 336-0911, Japan \\ Correspondence should be addressed to Kaoru Takeshima; pordoc1964@yahoo.co.jp
}

Received 25 May 2016; Revised 28 July 2016; Accepted 3 August 2016

Academic Editor: Paola De Nardi

Copyright ( 2016 Kaoru Takeshima et al. This is an open access article distributed under the Creative Commons Attribution License, which permits unrestricted use, distribution, and reproduction in any medium, provided the original work is properly cited.

\begin{abstract}
Isolated para-aortic lymph node (PLN) recurrence from colorectal cancer (CRC) is rare, with no currently validated treatments. Few reports have described the successful resection of isolated PLN involvement from CRC following chemotherapy. We report the case of a 63-year-old man who underwent sigmoidectomy for sigmoid colon cancer at our hospital. Pathological examination demonstrated advanced sigmoid colon cancer with metastatic involvement in both of the tested PLNs. Palliative chemotherapy was initiated four weeks after surgical resection, with administration of the FOLFIRI regimen. Four years after the operation, computed tomography (CT) revealed an enlarged PLN below the left renal vein. As PLN enlarged to $15 \mathrm{~mm}$ in the minor axis on a CT scan in 2014 after receiving a total of 156 courses of the FOLFIRI regimen, we considered the enlarged PLN to represent an isolated metastasis. Accordingly, lymph node resection was performed with microscopically negative margins. The patient maintained a good quality of life without any side effects throughout the whole course of his treatment and remains disease-free at 24 months without chemotherapy after resection of the isolated PLN. Curative resection following chemotherapy may improve survival of carefully selected advanced CRC patients with locoregional recurrence, such as isolated PLN involvement.
\end{abstract}

\section{Introduction}

Isolated para-aortic lymph node (PLN) recurrence of colorectal cancer (CRC) is rare and validated treatments have yet to be established; therefore, the prognosis of patients with isolated PLN recurrence is poor [1, 2]. Surgical resection for the local recurrence of colorectal cancer following chemotherapy is, in cases where complete resection is possible, the only modality shown to confer long-term survival $[1,3]$. Salvage surgery for isolated PLN recurrence is likely to improve the survival of patients with metastatic CRC [1]. Palliative chemotherapy comprising two key drugs-irinotecan and oxaliplatin-combined with leucovorin (LV) and 5fluorouracil (FU) for metastatic CRC has been shown to improve both progression-free survival and overall survival in patients with metastatic CRC [4]. Here we report the successful resection of isolated PLN recurrence in a patient who received a total of 156 courses of the FOLFIRI regimen following sigmoidectomy for sigmoid colon cancer.

\section{Case Presentation}

A 63-year-old man underwent sigmoidectomy for sigmoid colon cancer at our hospital in 2007. Histological examination of the surgical specimen revealed well-differentiated adenocarcinoma (Figure 1) that had invaded the subserosal layer (T3) with metastatic involvement in 12 of 20 dissected regional lymph nodes $(\mathrm{N} 2 \mathrm{~b})$ and in both of the sampled PLNs (Mla). The tumor was retrospectively staged as IVA according to the TNM classification [5]. As residual disease was detected microscopically, palliative chemotherapy was initiated four weeks after surgical resection, with administration of the FOLFIRI regimen consisting of a 120-minute infusion of $l$-leucovorin $200 \mathrm{mg} / \mathrm{m}^{2}$ and a 90 -minute infusion 


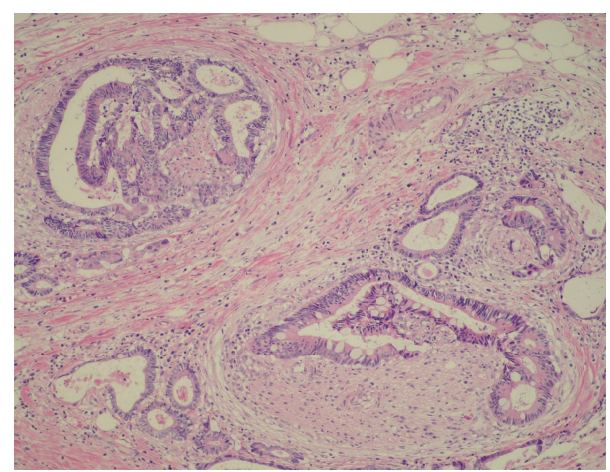

FIGURE 1: Histological examination of resected sigmoid colon cancer specimen. Histological examination revealed a well-differentiated adenocarcinoma. Hematoxylin and eosin stain (H\&E), low magnification.

of irinotecan $150 \mathrm{mg} / \mathrm{m}^{2}$ followed by a $400 \mathrm{mg} / \mathrm{m}^{2}$ bolus of 5 FU and a 46 -hour infusion of 5-FU $2400 \mathrm{mg} / \mathrm{m}^{2}$ every two weeks. Monoclonal agents were not combined for economic reasons. Computed tomography (CT) imaging was repeated every four months to assess the efficacy of chemotherapy. In 2011, following completion of the 92nd cycle of FOLFIRI, an abdominal CT scan demonstrated an enlarged PLN below the left renal vein (Figure 2(a)). Although we proposed positron emission tomography to diagnose whether the enlarged PLN was metastatic or not, he refused it. Under the informed consent of the patient, the FOLFIRI regimen has been continued since this time. The PLN was found to have enlarged to $15 \mathrm{~mm}$ in the minor axis on a CT scan performed in 2014 after the patient had completed a total of 156 courses of the FOLFIRI regimen (Figure 2(b)). The enlarged PLN was accordingly considered a metastatic at this time [6]. There was no elevation in his serum carcinoembryonic antigen levels throughout the course of the treatment. As there was no evidence of recurrence at any other sites on CT imaging; we elected to perform lymph node resection. Histological examination revealed involvement of the PLN by well-differentiated adenocarcinoma with negative margins, with similar histology to the resected sigmoid colon cancer (Figure 3 ). The patient did not suffer any adverse events, such as hematotoxicity or intestinal toxicity, at any time during the course of his treatment and has remained disease-free without chemotherapy for 24 months since resection of the isolated PLN, with no evidence of recurrence of sigmoid colon cancer (Figure 4).

\section{Discussion}

As the major sites of initial CRC metastases are the liver and lung; CRC metastases can commonly be resected. Curative resection of liver or lung metastases has been shown to improve survival in patients with metastatic CRC. Five-year survival rates of $30 \%-40 \%$ and $48 \%$ have been reported following the resection of liver and lung CRC metastases, respectively [7]. On the other hand, locoregional recurrence, which constitutes $10 \%-20 \%$ of all recurrences of CRC, is less common than recurrence at distant sites [8]; however, complete resection of locoregional recurrence has been shown to confer the same long-term survival in patients with liver or lung metastases [3]. Shibata et al. [9] and Bowne et al. [10] reported five-year median survival rates in patients with curative resection of locoregional recurrence from metastatic CRC of 81 months and 44 months, respectively. Moreover, the five-year survival rate of patients, who underwent curative resection of extraregional lymph node metastases including involvement of PLN from CRC, is significantly higher than that of patients who received palliative surgery $(70.3 \%$ versus $12.5 \%$ ) [11]. The incidence of isolated PLN recurrence is reportedly $1.3 \%$, with a median survival time of 34 months in patients with resection of isolated PLN involvement and just 14 months in patients without resection [1]. Bae et al. [12] reported five-year survival rates of $33.9 \%$ in patients with curative resection of isolated PLN recurrence. These reports indicate that curative resection with microscopic negative margins of isolated PLN recurrence may improve the survival of patients with isolated PLN involvement. In the present case, salvage surgery with no residual tumor was likely to have contributed to the long-term survival of this patient.

Palliative chemotherapy comprising two key drugsoxaliplatin and irinotecan-has demonstrated efficacy in improving the survival of patients with metastatic CRC [4]. Furthermore, the combination of monoclonal agents against angiogenesis or epidermal growth factor receptor (EGFR) has been shown to prolong overall survival better than that by chemotherapy alone [7]. In the present case report, the patient received FOLFIRI as a first-line regimen following sigmoid colon resection as a small bowel obstruction can develop due to peritoneal carcinomatosis from PLN involvement. A number of retrospective studies have reported that multidisciplinary therapy can improve the prognosis of patients with isolated PLN recurrence. Min et al. [1] reported a median survival time after recurrence of 14 months in 32 patients who received chemotherapy without resection of isolated PLN recurrence of whom 19 patients underwent radiation therapy plus concurrent or sequential chemotherapy. A median survival time of 37 months was reported in seven patients with isolated PLN recurrence from CRC who underwent stereotactic radiation therapy, with a median recurrence-free survival time of 26 months [2]. The major limitations of these reports are small sizes and retrospective nature of the studies. However, chemotherapy, radiation therapy, or chemoradiation therapy are likely to have efficacy in improving the overall survival time in patients with isolated PLN recurrence from CRC. Yasuda et al. [13] reported an interesting case of a patient with isolated PLN recurrence from CRC who has survived for 6 years with complete remission after chemoradiation therapy.

There are three major factors to consider regarding the treatment of cases such as the ones discussed in this study. First, there is a controversy regarding the use of immediate treatment or expectant management in patients with microscopic residual but asymptomatic disease following resection. A recent study reported that early treatment resulted in significantly prolonging the survival of asymptomatic patients with advanced CRC [14]. Conversely, a meta-analysis found no significant improvement in survival with early 


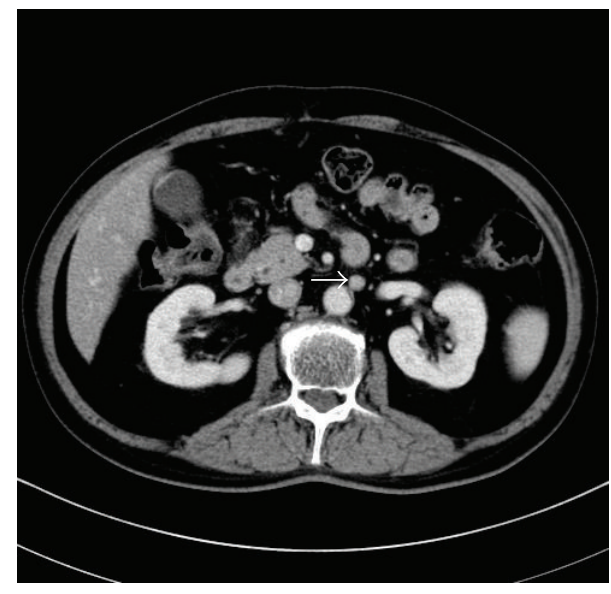

(a)

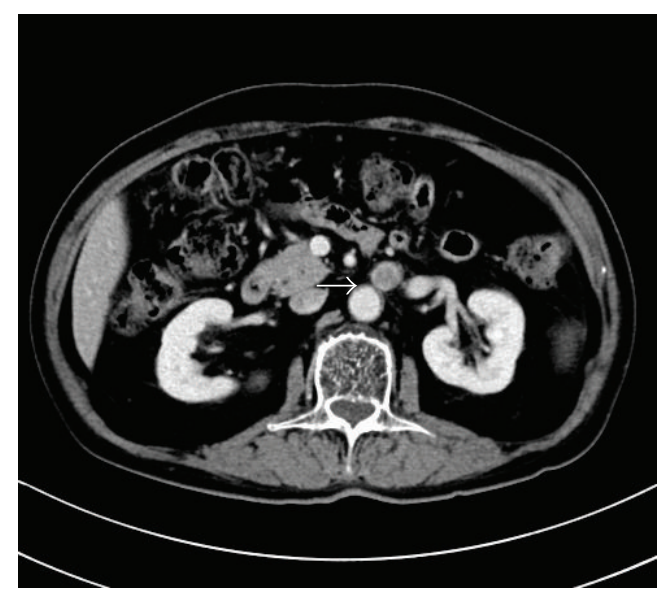

(b)

FIGURE 2: CT imaging of an enlarged para-aortic lymph node. Abdominal CT demonstrated an enlarged isolated para-aortic lymph node (a) in 2011. The lymph node was found to have enlarged to $15 \mathrm{~mm}$ in the minor axis (b) in 2014.

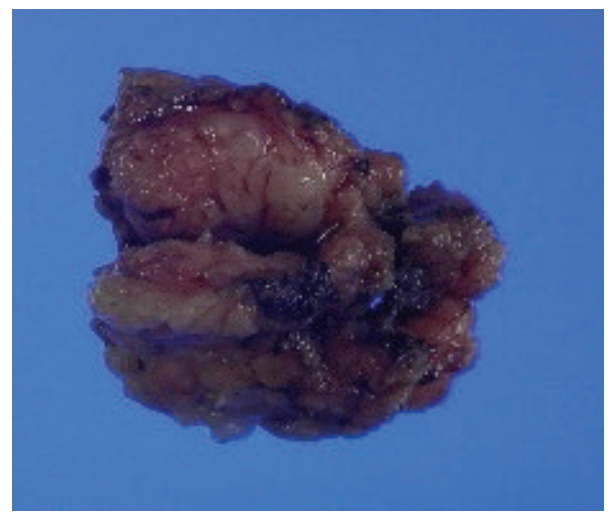

(a)

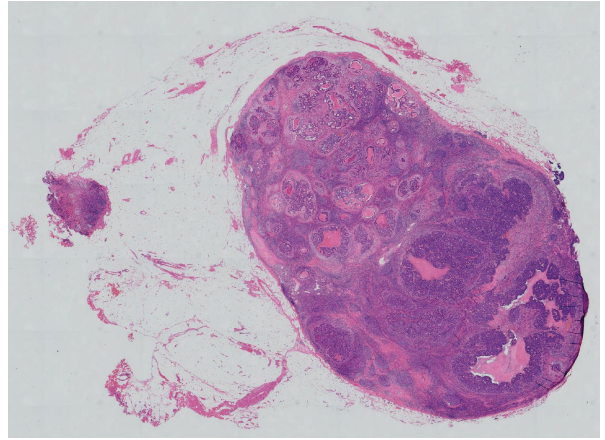

(b)

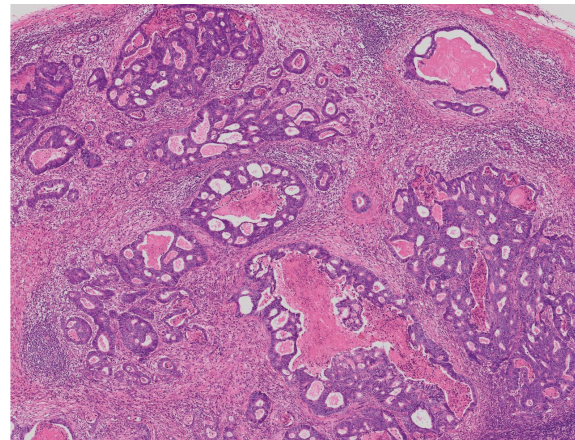

(c)

FIGURE 3: Histological examinations of the resected para-aortic lymph node. Histological examination revealed that the para-aortic lymph node was involved by well-differentiated adenocarcinoma with negative margins, with similar histology to the resected sigmoid colon cancer specimen. (a) Resected specimen, (b) H\&E, original magnification, and (c) H\&E, low magnification.

treatment of asymptomatic patients with advanced CRC [15]. In the present case, early treatment with FOLFIRI allowed successful resection of isolated PLN recurrence from CRC. Second, the use of intermittent or continuous immediate treatment remains contentious. No significant difference in the overall survival of patients with advanced CRC has been demonstrated between intermittent FOLFIRI and continuous FOLFIRI; however, the patients who underwent intermittent FOLFIRI are reportedly at a decreased risk of venous thromboembolism [16]. In the present case, FOLFIRI was immediately initiated and continued until 2014 when the patient underwent resection of an isolated PLN recurrence 


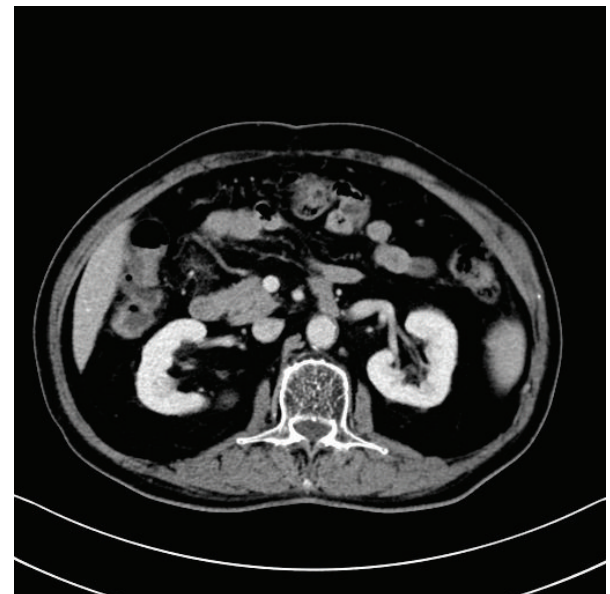

Figure 4: CT imaging performed in 2016. CT scan showed no evidence of para-aortic lymph node recurrence.

without any toxicities or adverse events. Third, the use of postoperative chemotherapy after the resection of PLN recurrence is controversial. Currently, as far as we know, there is no evidence that postoperative chemotherapy improves overall survival in patients with microscopically resected CRC recurrence [17]. In the present case, the patient has remained disease-free without chemotherapy for 24 months after resection of PLN recurrence.

Over a six-year period, our patient received a total of 156 continuous courses of the FOLFIRI regimen without any dose reduction or any pause and maintained a good quality of life with almost stable disease without any adverse events. To our knowledge, there have been no previous reports describing the long-term use of the FOLFIRI regimen as in our case, although a number of studies have reported that monoclonal antibody agents against angiogenesis or EGFR can be tolerated in long-term use $[18,19]$. The long-term use of FOLFIRI was possible in our case for two reasons. First, it was indicated that, given the lack of adverse events during the course of the treatment, the patient's UGT1A haplotype contained neither UGT1A1* 6 nor UGT1A1 ${ }^{*} 28$ [20]. Second, the cumulative toxicity, such as neurotoxicity from the cumulative use of FOLFOX, is rarely associated with that of FOLFIRI [4].

\section{Conclusion}

Here we report the successful resection of isolated PLN recurrence in a case of advanced sigmoid colon cancer following a total of 156 courses of the FOLFIRI regimen. Curative resection following chemotherapy may improve the survival of carefully selected advanced CRC patients with locoregional recurrence, such as isolated PLN involvement. The FOLFIRI regimen may be well tolerated in long-term use.

\section{Consent}

Written informed consent was obtained from the patient for publication of this case report and any accompanying images. A copy of the written consent is available for review.

\section{Competing Interests}

The authors declare that there is no conflict of interests regarding the publication of this paper.

\section{Authors' Contributions}

Kaoru Takeshima performed the operation and chemotherapy. Hideo Baba gave suggestions regarding chemotherapy indication. Kaoru Takeshima drafted the paper, and all authors revised the paper. All authors read and approved the final paper.

\section{References}

[1] B. S. Min, N. K. Kim, S. K. Sohn, C. H. Cho, K. Y. Lee, and S. H. Baik, "Isolated paraaortic lymph-node recurrence after the curative resection of colorectal carcinoma," Journal of Surgical Oncology, vol. 97, no. 2, pp. 136-140, 2008.

[2] M.-S. Kim, C. K. Cho, K. M. Yang, D. H. Lee, S. M. Moon, and Y. J. Shin, "Stereotactic body radiotherapy for isolated paraaortic lymph node recurrence from colorectal cancer," World Journal of Gastroenterology, vol. 15, no. 48, pp. 6091-6095, 2009.

[3] R. G. Landmann and M. R. Weiser, "Surgical management of locally advanced and locally recurrent colon cancer," Clinics in Colon and Rectal Surgery, vol. 18, no. 3, pp. 182-189, 2005.

[4] C. Tournigand, T. André, E. Achille et al., "FOLFIRI followed by FOLFOX6 or the reverse sequence in advanced colorectal cancer: a randomized GERCOR study," Journal of Clinical Oncology, vol. 22, no. 2, pp. 229-237, 2004.

[5] L. H. Sobin, M. K. Gospodarowicz, and C. Wittekind, International Union against Cancer: Tnm Classification of Malignant Tumours, Union for International Cancer Control, West Sussex, UK, 7th edition, 2010.

[6] E. A. Eisenhauer, P. Therasse, J. Bogaerts et al., "New response evaluation criteria in solid tumours: revised RECIST guideline (version 1.1)," European Journal of Cancer, vol. 45, no. 2, pp. $228-$ 247, 2009.

[7] I. Chau and D. Cunningham, "Treatment in advanced colorectal cancer: what, when and how?" British Journal of Cancer, vol. 100, no. 11, pp. 1704-1719, 2009.

[8] C. J. H. van de Velde, P. G. Boelens, J. M. Borras et al., "EURECCA colorectal: multidisciplinary management: European consensus conference colon \& rectum," European Journal of Cancer, vol. 50, no. 1, pp. 1.el-1.e34, 2014.

[9] D. Shibata, P. B. Paty, J. G. Guillem, W. D. Wong, and A. M. Cohen, "Surgical management of isolated retroperitoneal recurrences of colorectal carcinoma," Diseases of the Colon and Rectum, vol. 45, no. 6, pp. 795-801, 2002.

[10] W. B. Bowne, B. Lee, W. D. Wong et al., "Operative salvage for locoregional recurrent colon cancer after curative resection: an analysis of 100 cases," Diseases of the Colon and Rectum, vol. 48, no. 5, pp. 897-909, 2005.

[11] A. Ogura, T. Akiyoshi, Y. Takatsu et al., "The significance of extended lymphadenectomy for colorectal cancer with isolated synchronous extraregional lymph node metastasis," Asian Journal of Surgery, 2015.

[12] S. U. Bae, Y. D. Han, M. S. Cho et al., "Oncologic outcomes of colon cancer patients with extraregional lymph node metastasis: comparison of isolated paraaortic lymph node metastasis 
with resectable liver metastasis," Annals of Surgical Oncology, vol. 23, no. 5, pp. 1562-1568, 2016.

[13] S. Yasuda, H. Kamata, T. Machida et al., "A case of isolated paraaortic lymph node recurrence from colon cancer successfully treated with chemoradiotherapy," The Tokai Journal of Experimental and Clinical Medicine, vol. 37, no. 2, pp. 47-50, 2012.

[14] Nordic Gastrointestinal Tumor Adjuvant Therapy Group, "Expectancy or primary chemotherapy in patients with advanced asymptomatic colorectal cancer: a randomized trial," Journal of Clinical Oncology, vol. 10, no. 6, pp. 904-911, 1992.

[15] S. P. Ackland, M. Jones, D. Tu et al., "A meta-analysis of two randomised trials of early chemotherapy in asymptomatic metastatic colorectal cancer," British Journal of Cancer, vol. 93, no. 11, pp. 1236-1243, 2005.

[16] M. Mandalà, S. Barni, I. Floriani et al., "Incidence and clinical implications of venous thromboembolism in advanced colorectal cancer patients: the 'GISCAD-alternating schedule' study findings,' European Journal of Cancer, vol. 45, no. 1, pp. 65-73, 2009.

[17] R. Araujo, M. Gonen, P. Allen et al., "Comparison between perioperative and postoperative chemotherapy after potentially curative hepatic resection for metastatic colorectal cancer," Annals of Surgical Oncology, vol. 20, no. 13, pp. 4312-4321, 2013.

[18] H. I. Hurwitz, W. Honeycutt, S. Haley, and J. Favaro, "Longterm treatment with bevacizumab for patients with metastatic colorectal cancer: case report," Clinical Colorectal Cancer, vol. 6, no. 1, pp. 66-69, 2006.

[19] E. Seront, L. Marot, E. Coche, J.-L. Gala, C. Sempoux, and Y. Humblet, "Successful long-term management of a patient with late-stage metastatic colorectal cancer treated with panitumumab," Cancer Treatment Reviews, vol. 36, supplement 1, pp. S11-S14, 2010.

[20] H. Minami, K. Sai, M. Saeki et al., "Irinotecan pharmacokinetics/pharmacodynamics and UGT1A genetic polymorphisms in Japanese: roles of UGT1A1 ${ }^{\star} 6$ and ${ }^{*} 28$," Pharmacogenetics and Genomics, vol. 17, no. 7, pp. 497-504, 2007. 


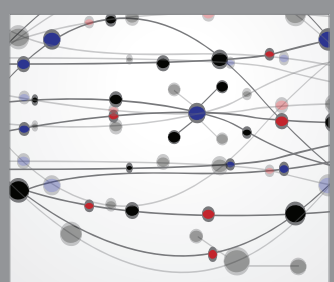

The Scientific World Journal
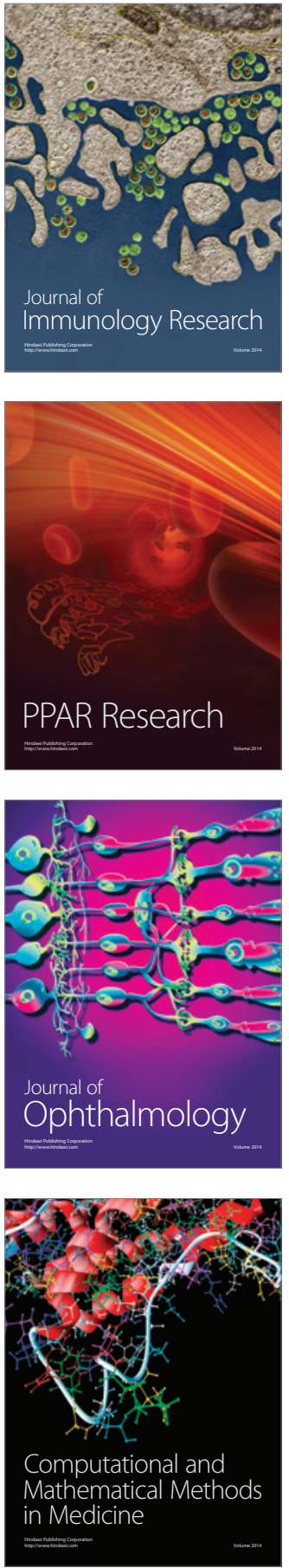

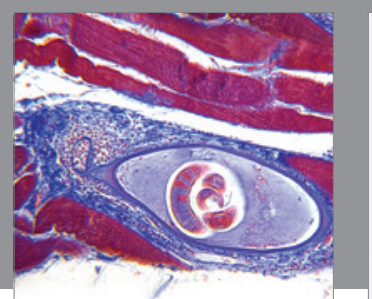

Gastroenterology Research and Practice

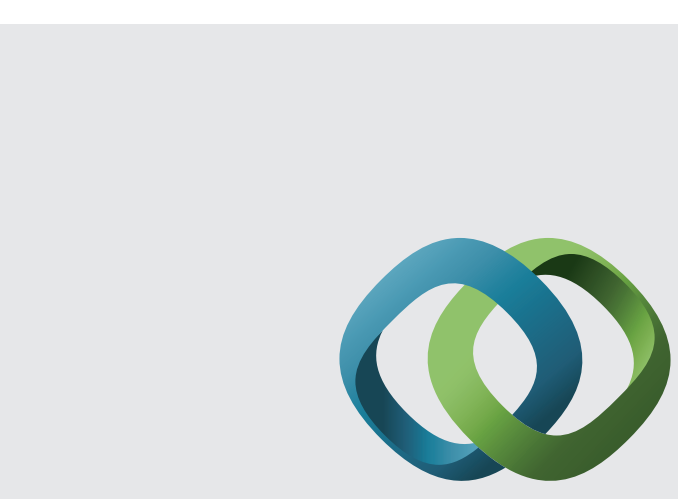

\section{Hindawi}

Submit your manuscripts at

http://www.hindawi.com
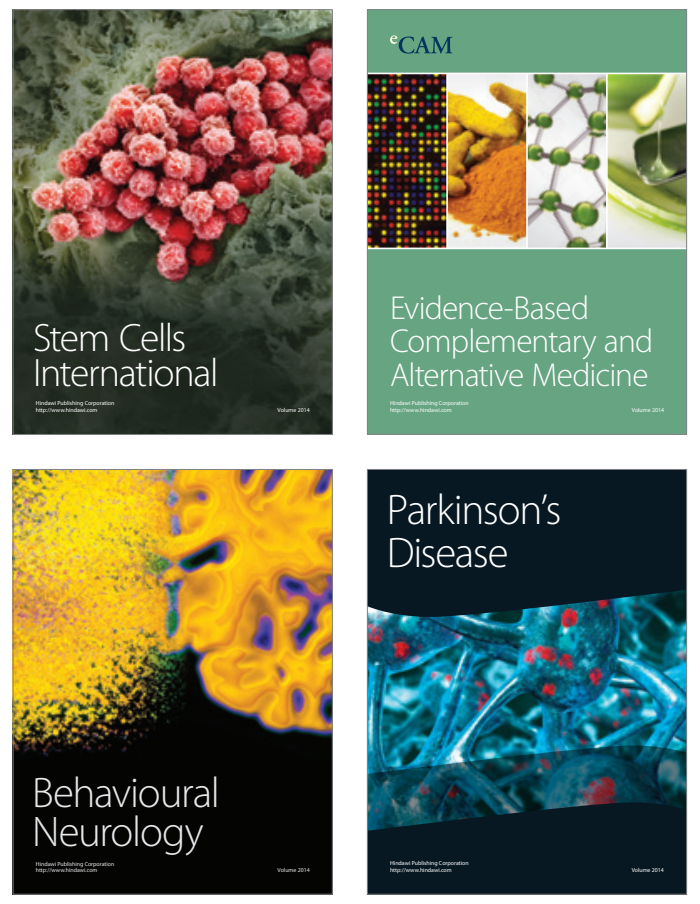
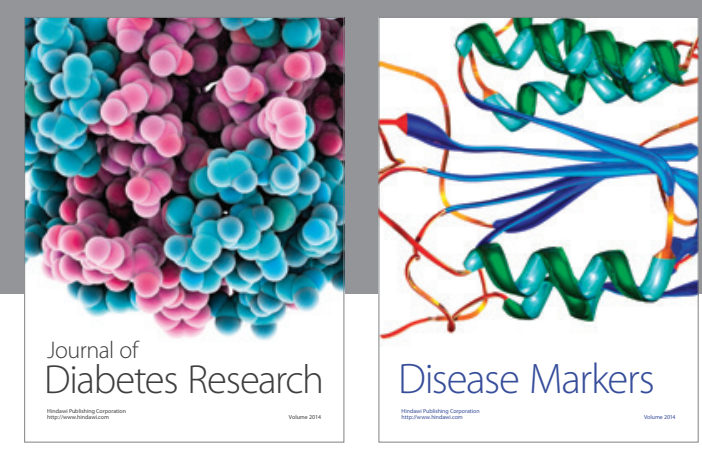

Disease Markers
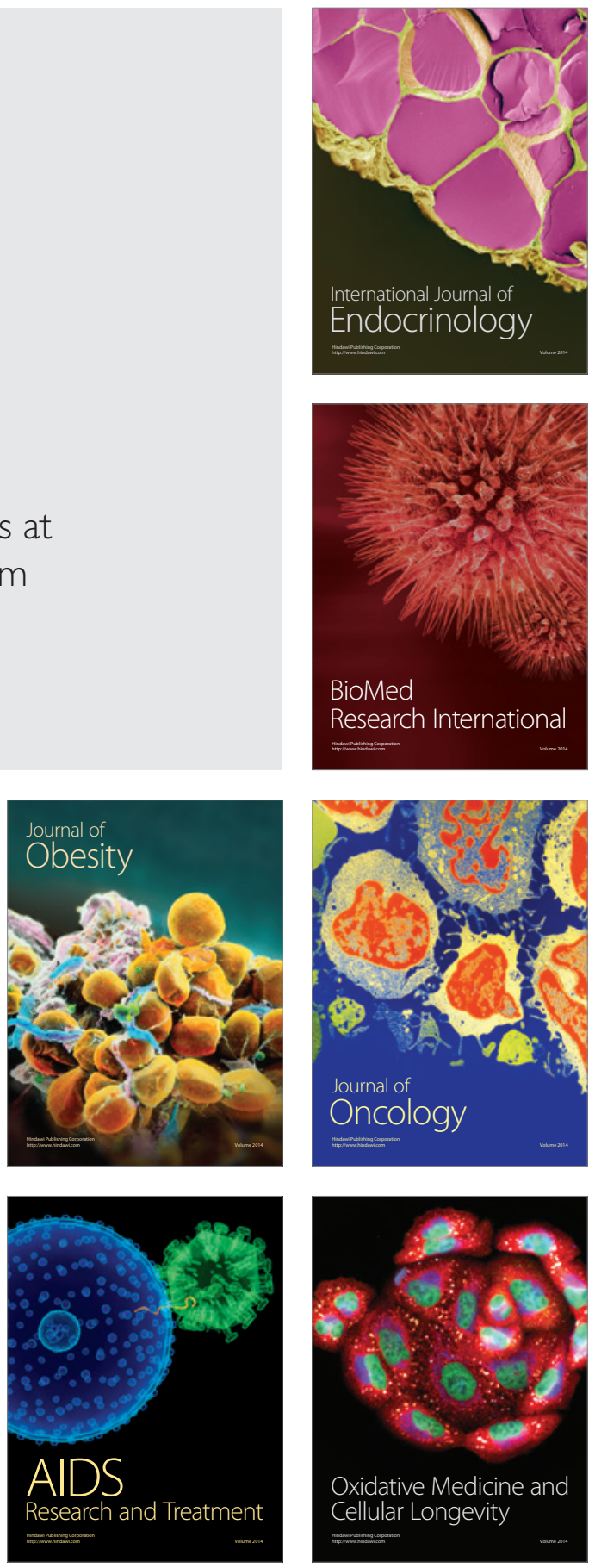